\title{
Clinical Feasibility of Interactive Commercial Nintendo Gaming for Chronic Stroke Rehabilitation
}

\author{
Eun Kyung Kim, MS, $\mathrm{PT}^{1)}$, Jong Ho Kang, $\mathrm{PhD}, \mathrm{PT}^{2)}$, \\ Jang Sung Park, PhD, $\mathrm{PT}^{1)}$, Byung Ho Jung ${ }^{3)}$ \\ 1) Department of Physical Therapy, Seonam University \\ 2) Department of Physical Therapy, College of Health Sciences, Catholic University of Pusan: 57 \\ Oryundae-ro, Geumjeong-gu, Busan, 609-757 Republic of Korea. \\ TEL: +82 51-510-0572,FAX: +82 51-510-0578,E-mail: swithun@cup.ac.kr \\ 3) Department of Biomedical Engineering, Nambu University
}

\begin{abstract}
Purpose] The purpose of this study was to investigate the effect of interactive games played on a Nintendo Wii on postural control, motor function and functional independence of chronic stroke patients. [Subjects and Methods] Twenty chronic stroke patients were recruited for this study. The subjects were randomly divided into experimental and control groups. The experimental group played interactive games using the Nintendo Wii for 30 minutes a day, 3 times a week, for 3 weeks, whereas the control group did not play interactive games. Result measures included the postural assessment scale, the modified motor assessment scale and the functional independence measure. [Results] There were significant differences in the postural assessment scale and modified motor assessment scale scores of both groups after the intervention, and between the control group and the experimental group after the intervention. However, there were no significant differences in functional independence measure scores. [Conclusion] An interactive game played on a Nintendo Wii appears to be an effective intervention for improvement of chronic stroke patients' functional results. Therefore, research into the most effective type of Wii game and the clinical significance should continue.

Key words: Nintendo Wii, Interactive game, Chronic stroke
\end{abstract}

(This article was submitted Apr. 9, 2012, and was accepted May 8, 2012)

\section{INTRODUCTION}

Rehabilitation programs in which chronic stroke patients can easily participate after discharge from the hospital are needed. Recent studies have placed emphasis on intensive and repetitive training, motor learning utilizing taskspecific movement, and visual and auditory feedback rather than passive approaches. Furthermore, the necessity of active and positive participation accompanied by fun and self-motivation has been emphasised ${ }^{1-4)}$. Therefore, new rehabilitation programs should reflect this in the methods they use to treat patients.

Today, virtual reality that embodies the real environment, activities, and movements are known to be effective for rehabilitation, and a variety of virtual reality programs are now being studied for use in the rehabilitation of neurological patients ${ }^{5,6)}$. Virtual reality rehabilitation programs have diverse tasks and goals, which can be easily adjusted for degrees of difficulty in activities, are fun, and effectively employ visual and auditory feedbacks ${ }^{7)}$. However, previous studies have mostly used expensive equipment and specialized programs, making it difficult for chronic stroke patients to take part in such programs after discharge from hospital.
Recently, interactive gaming that is fun has started to be introduced into the rehabilitation field. The most representative tool is the Nintendo Wii (NW), whose commercial distribution began in 2006. Its wireless remote control enables play of virtual sports games by measuring the direction and acceleration of the upper extremity and trunk movements. Moreover, NW games are interactive, with visual and auditory feedbacks. They provide fun and scores as results, improving players sports performance. NW games motivate patients, enabling them to have fun, encouraging their active participation in the repetition of goal-oriented and task-specific movements ${ }^{8)}$. Nevertheless, relevant research into their clinical applications has been lacking. Accordingly, this study examined the feasibility of using commercially available NW games that are easy to purchase at low cost, and can be performed with safety in the rehabilitation of stroke patients, by examining the effect of their performance on postural control and motor function, and determined the feasibility of their use for rehabilitation of stroke patients.

\section{SUBJECTS AND METHODS}

This study's subjects were 20 stroke patients with 
functional impairment. The participants were inpatients who were diagnosed with stroke by magnetic resonance imaging (MRI) or Computed Tomography (CT) more than 6 months earlier. All the subjects scored 19 or higher points on the mini-mental status examination (MMSE) and were able to maintain an upright posture without any assistance. Those who had received orthopedic surgery or had a history of arthritis, hand or upper limb pain, epilepsy, or psychiatric illnesses were excluded from this study. Those who had played a virtual reality game before were also excluded. Twenty patients were randomly allocated to either the experimental group or the control group, a total of 10 members per group. Three patients dropped out of the experiment due to discharge, recurrence of stroke, and injury from a fall. The number of subjects who completed the intervention was 10 in the experimental group and 7 in the control group. The experimental group's average age, height, weight, onset time, and MMSE-K score were $41.30 \pm 6.61$ years, 165.10 $\pm 8.86 \mathrm{~cm}, 65.40 \pm 10.59 \mathrm{~kg}, 12.6 \pm 7.12$ months, and 24.8 \pm 1.98 , respectively. The control group's average age, height, weight, onset time, and MMSE-K score were 55.00 \pm 13.02 years, $161.90 \pm 6.09 \mathrm{~cm}, 62.84 \pm 12.78 \mathrm{~kg}, 12.85$ \pm 6.06 months, and $25.14 \pm 2.1 .9$, respectively. The types of stoke were hemorrhage (experimental group 4, control group 3) and infarction (experimental group 6, control group 4).

The 10 patients in the experimental group played Nintendo Wii games (Nintendo Inc., Japan), while the 10 patients in the control group did not. A 30-inch TV set placed at the height of $60 \mathrm{~cm}$ from the ground was used and the motion sensor was mounted on the TV set. The distance between the sensor and the remote control was set at 1.5 meters. Because the patients had different physical characteristics, the distance between the remote control and the sensor was adjusted for each individual prior to exercise. The subjects in the experimental group played the game by holding the Wii remote and Nunchuk controllers. Some participants who had difficulty in holding the controllers played the game with fabric grasp assistance or by strapping them to their hands with a thin bandage. All the games were performed with the players maintaining an upright posture. Tennis games were played by the patients with the remote control held in the hand on the normal side, and boxing games were played with the remote control held in the hand on the normal side and the Nunchuk controller in the hand of the paralyzed side. The game software used in this study was Wii Sports (Nintendo Inc., Japan). All the games provided by the software require motions in different directions and with differing acceleration. To control these differences, the games played were confined to certain games for consistency. The patients were informed of their game scores. Knowledge of scores motivated subjects in their task, encouraging greater effort to obtain higher scores, and active participation. The exercise was carried out 3 times per week for 3 weeks, a total of 9 sessions. The term of each session was 30 minutes: 15 minutes of the tennis game and 15 minutes of the boxing game. Prior to their participation in the gaming exercise, all the subjects in the experimental and control groups received general exercise for 30 minutes and electrical stimulation of the tibialis anterior on affected side for 15 minutes.

The results were measured prior to the intervention and after three weeks of the intervention. The tools used in the evaluation were the postural assessment scale (PASS), for the assessment of postural control ability of stroke (scores of 0-36), the modified motor assessment scale (MMAS), for the assessment of motor ability (scores of $0-54$ ), and the functional independence measure (FIM), for the assessment of functional independence (scores of 18-126). Data obtained in the experiment are reported as mean and standard deviation values. In order to examine at the differences in PASS, MMAS, and FIM scores of the control and the experimental groups before and after the intervention, the paired t-test was conducted, and in order to examine whether there were differences in the post-intervention scores between the control and the experimental group, analysis of covariance (ANCOVA) was conducted on the covariance calculated from the pre-intervention scores. A probability value of $\mathrm{p}<0.05$ was considered to be statistically significant.

\section{RESULTS}

Significant increases in the PASS and MMAS scores in both the control and the experimental groups after the intervention were observed $(p<0.05)$ (Table 1). Significant differences in the PASS and MMAS scores between the control group and the experimental group after the intervention were also observed $(p<0.05)$ (Table 2$)$. However, there were no significant differences in the FIM scores in the control or experimental groups before and after the intervention ( $p>0.05)$ (Table 1), and no significant difference in FIM scores between the experimental group and the control group $(\mathrm{p}>0.05)$ (Table 2$)$.

\section{DISCUSSION}

Despite several limitations, exercise using virtual reality software is considered to be very effective. In the past, most research into rehabilitation based on virtual reality has been conducted on programs that represent actual activities ${ }^{9)}$, but recently researchers are looking into programs in the form of games $^{10)}$. The NW gaming exercise conducted for 3 weeks in a total of 9 sessions in this study enhanced chronic stroke patients' abilities to adjust posture and motor function, and their extent of recovery was significantly greater than that of the control group. In spite of this study's small sample size, its result, obtained with a controlled experimental method, shows the possibility of an economical and safe new management method for stroke patients as their performance of the NW gaming exercise resulted in significant improvement in their conditions. Our results are consistent with those of Merians et al. ${ }^{11)}$, who reported that repetitive and intensive performance of a virtual reality program led to improved upper extremity function. An advantage of NW gaming is that although chronic stroke patients cannot perform sports such as tennis or boxing, they can reproduce sports motions in virtual reality. This aspect of NW gaming imbues chronic stroke patients with self-confidence, and encourages their active participation, enabling repetitive and 
Table 1. Comparison of PASS, MMAS and FIM scores pre- and post-intervention within each group

\begin{tabular}{cccc}
\hline \multicolumn{2}{c}{ Category } & Pre-intervention & Post-intervention \\
\hline Control & PASS* & $26.42 \pm 1.98$ & $28.00 \pm 1.63$ \\
group & MMAS* & $31.71 \pm 1.88$ & $33.57 \pm 1.51$ \\
& FIM & $101.00 \pm 7.89$ & $101.28 \pm 8.11$ \\
Experiment & PASS* & $25.80 \pm 3.85$ & $30.30 \pm 3.19$ \\
group & MMAS* & $29.80 \pm 6.40$ & $34.70 \pm 6.20$ \\
& FIM & $101.50 \pm 13.64$ & $103.3 \pm 4.32$ \\
\hline
\end{tabular}

* Significant difference $(\mathrm{p}<0.05)$. Unit: score

Table 2. Comparison of PASS, MMAS, and FIM scores between the experimental group and control group after the intervention

\begin{tabular}{lcc}
\hline Category & Control group & Experimental group \\
\hline PASS* & $28.00 \pm 1.63$ & $30.30 \pm 3.19$ \\
MMAS* & $33.57 \pm 1.51$ & $34.70 \pm 6.20$ \\
FIM & $101.28 \pm 8.11$ & $103.3 \pm 4.32$ \\
\hline
\end{tabular}

* Significant difference $(\mathrm{p}<0.05)$. Unit: score

intensive gaming exercise.

Visual, auditory, and tactile feedback prompts neurological patients' functional recovery ${ }^{4}$. The NW games reinforced patients' visual, auditory, and tactile feedback in all situations occurring during their gaming exercise through the screen, speaker, and remote control. The feedback enhanced patients' self-awareness, and they were able to correct diverse errors occurring during the exercise immediately, thereby improving their abilities to perform tasks. Patients obtained improved functional outcomes through the feedback provided by the NW games.

In this study, there was no significant difference in FIM scores between the experimental group and the control groups. This result is considered to be due to the characteristics of the evaluation scales. PASS evaluates posture maintenance and transformation and MMAS largely assess posture transformation, balance, upper limb functions, and muscle tension. However, FIM assess self-management, evacuation control, movement, communication, and perception. The NW gaming exercise used in this study mainly requires posture maintenance, transformation, balance, and upper limb functions. Our PASS and MMAS results are similar to those of Yong Joo et al. ${ }^{8)}$, who reported improvement in FMA scores, and Nitz et al. ${ }^{12)}$, who reported enhanced balance abilities. The control group's PASS and MMAS scores increased significantly. This is probably because the 30 -minute general exercise and application of electrical stimulation to the lower limbs, which were provided to all subjects, were effective. However, the experimental group's scores increased much more than those of the control group.

In this study, there were no adverse effects of the NW games. An experienced physical therapist supervised the gaming, standing close by in order to safeguard against loss of balance due to extreme motions. None of the ten participants fell or incurred injury. However, some patients who concentrated on the game excessively experienced loss of balance. Therefore, when conducting NW games for stroke patients, appropriate supervision is necessary. In conclusion, the NW games, which are more economical and easier to use than existing virtual reality tools, will be an effective tool for stroke patients, maintaining their posture and improving their functional results. Therefore, the development of programs using NW, research on them, and their clinical application should continue.

\section{REFERENCES}

1) Dobkin BH: Training and exercise to drive poststroke recovery. Nat Clin Pract Neurol, 2008, 4: 76-85. [Medline] [CrossRef]

2) Maclean N, Pound P, Wolfe C, et al.: Qualitative analysis of stroke patients' motivation for rehabilitation. BMJ, 2000, 321: 1051-1054. [Medline] [CrossRef]

3) Bisson E, Contant B, Sveistrup H, et al.: Functional balance and dual-task reaction times in older adults are improved by virtual reality and biofeedback training. Cyberpsychol Behav, 2007, 10: 16-23. [Medline] [CrossRef]

4) Zijlstra A, Mancini M, Chiari L, et al.: Biofeedback for training balance and mobility tasks in older populations: a systematic review. J Neuroeng Rehabil, 2010, 7. [Medline] [CrossRef]

5) Holden M, Todorov E, Callahan J, et al.: Virtual environment training improves motor performance in two patients with stroke. Case Rep Neurol Rep, 1999, 23: 56-67.

6) Jang SH, You SH, Hallett M, et al.: Cortical reorganization and associated functional motor recovery after virtual reality in patients with chronic stroke: an experimenter-blind preliminary study. Arch Phys Med Rehabil, 2005, 86: 2218-2223. [Medline] [CrossRef]

7) Rizzo AA, Kim GJ: A SWOT analysis of the field of VR rehabilitation and therapy. Presence Teleoperators Virtual Environ, 2005, 14: 119-146. [CrossRef]

8) Yong Joo L, Soon Yin T, Xu D, et al.: A feasibility study using interactive commercial off-the-shelf computer gaming in upper limb rehabilitation in patients after stroke. J Rehabil Med, 2010, 42: 437-441. [Medline] [CrossRef]

9) Christiansen C, Abreu B, Huffman RK: Creating a virtual environment for brian injury rehabilitation and research: a preliminary report. J Med Virtual Real, 1996, 1: 6-9.

10) Broeren J, Claesson L, Goude D, et al.: Virtual rehabilitation in an activity centre for community-dwelling persons with stroke. The possibilities of 3-dimensional computer games. Cerebrovasc Dis, 2008, 26: 289-296. [Medline] [CrossRef]

11) Merians AS, Jack D, Boian $\mathrm{R}$, et al.: Virtual reality-augmented rehabilitation for patients following stroke. Phys Ther, 2002, 82: 898-915. [Medline]

12) Nitz JC, Kuys S, Isles R, et al.: Is the Wii Fit a new-generation tool for improving balance, health and well-being? A pilot study. Climacteric, 2010, 13: 487-491. [Medline] [CrossRef] 